\title{
REFLECTION GROUPS AND CAT(0) COMPLEXES WITH EXOTIC LOCAL STRUCTURES
}

\author{
MICHAEL W. DAVIS AND JOHN MEIER
}

\begin{abstract}
We show that, in contrast to the situation for the standard complex on which a right angled Coxeter group $W$ acts, there are cocompact $W$-actions on CAT(0) complexes such that the local topology of the complex is distinctly different from the end topology of $W$.
\end{abstract}

If $\widetilde{X}$ is a contractible $n$-manifold or homology $n$-manifold, then, since it satisfies Poincaré duality, its cohomology with compact supports, $H_{c}^{*}(\widetilde{X})$, is concentrated in dimension $n$ and is isomorphic to $\mathbb{Z}$ in that dimension. It follows that the homology at infinity of $\widetilde{X}$ is concentrated in dimension $n-1$ and is isomorphic to $\mathbb{Z}$ in that dimension (see [?] for definitions and references for homology at infinity). More particularly, recall that a simplicial complex is a homology $n$-manifold if and only if the link of each vertex is a "generalized homology $(n-1)$-sphere" (i.e., a homology $(n-1)$-manifold with the same homology as $\left.S^{n-1}\right)$. So, the above argument shows that if the link of each vertex in an aspherical simplicial complex $X$ is a generalized homology $(n-1)$-sphere, then the homology at infinity of its universal cover $\widetilde{X}$ is concentrated in dimension $n-1$ and hence, $\tilde{X}$ is $(n-2)$-acyclic at infinity. This shows that hypotheses concerning the local topology of an aspherical space can have implications for the end topology of its universal cover.

There are other local-to-asymptotic results for nonpositively curved complexes. If $L$ is a simplicial complex, we let $\mathcal{S}(L)$ denote the set of all closed simplices of $L$, including the empty simplex. Let $\widetilde{X}$ be a (locally finite) CAT(0) cubical complex, and for each vertex $x \in \tilde{X}$, let $L_{x}$ denote its link. If for each vertex $x$ and for each closed simplex $\sigma \in \mathcal{S}\left(L_{x}\right), L_{x}-\sigma$ is $m$-connected (resp., $m$-acyclic), then $\widetilde{X}$ is $m$-connected (resp., $m$-acyclic) at infinity (see [?] and the references cited there). We call the complexes $L_{x}-\sigma$ the punctured links of $\widetilde{X}$.

In recent work [?], we have shown there is a close connection between local topology and end topology of the standard complexes on which Coxeter groups act. The nerve of a Coxeter system $(W, S)$ is the simplicial complex $L$ with one vertex for each element of the generating set $S$ and one simplex for each subset of $S$ which generates a finite subgroup of $W$. As explained in [?], [?] or [?], associated to $(W, S)$ there is natural cell complex, here denoted $|W|$, such that $|W|$ is a model for $\mathrm{E} W$ and such that the link of each of its vertices is isomorphic to $L$ (see [?] for the definition of $\mathrm{E} G$ ). This implies, for example, that if $L$ is a triangulation of an $(n-1)$-sphere, then $|W|$ is a contractible $n$-manifold.

Date: January 15, 2004.

Key words and phrases. Coxeter groups, topology at infinity .

MSC 2000: 20F55, 20J05, 51E24, 57M07.

Davis was partially supported by NSF grant DMS-0104026.

Meier thanks The Ohio State University for hosting him while on sabbatical. 
If $S$ is finite (which we shall henceforth always assume), then $L$ is a finite complex and the quotient space $|W| / W$ is compact. It is proved in [?] and [?] that the natural piecewise Euclidean metric on $|W|$ is CAT(0). In [?] the authors established a direct correspondence between the topological properties of $L$ and the asymptotic topological properties of $|W|$ (or of any locally finite building with associated Coxeter system $(W, S))$. For example:

Theorem. (See 4.1, 4.2 and 4.3 in [?]) Let $W$ be a finitely generated Coxeter group with associated nerve $L$. Then

(1) $W$ is simply connected at infinity if and only if $L-\sigma$ is simply connected for each $\sigma \in \mathcal{S}(L)$.

(2) $W$ is $m$-acyclic at infinity if and only if $L-\sigma$ is $m$-acyclic for each $\sigma \in \mathcal{S}(L)$.

(3) $W$ is $m$-connected at infinity if and only if $L-\sigma$ is $m$-connected for each $\sigma \in \mathcal{S}(L)$

Thus, not only do the connectivity properties of the punctured links $L-\sigma$ determine the connectivity at infinity of $\mathrm{W}$ (i.e., of $|W|$ ), the converse is also true. This leads to speculation that, in the general context of nonpositive curvature, similar asymptotic-to-local results might hold. For example, one might speculate that if $X$ is a nonpositively curved, finite Poincaré complex with, say, extendable geodesics, then the links of vertices in $X$ are forced to be generalized homology spheres (and hence, $X$ is a homology manifold). Similarly, it could be speculated that if $X$ is a nonpositively curved cubical complex (with extendable geodesics) and if $\widetilde{X}$ is $m$-connected (resp., $m$-acyclic) at infinity, then the punctured links of vertices must be $m$-connected (resp., $m$-acyclic). The purpose of this note is to give some examples which set such speculations to rest: there are no general results of this nature. (For further examples illuminating the difficulty of getting asymptotic-to-local results, see [?].)

The construction of our examples is essentially the same as the construction of [?]. We will show that by making minor modifications in the construction of $|W|$ one gets a model for $\underline{\mathrm{E}} W, \mathfrak{W}$, with a $\mathrm{CAT}(0)$ cubical structure so that the connectivity properties at infinity do not descend to connectivity properties of links. In fact, in all of our examples $|W|$ will be a manifold while $\mathfrak{W}$ will not even be a homology manifold. We show that $|W|$ and $\mathfrak{W}$ are equivariantly proper homotopy equivalent, so if $\Gamma$ is any torsion-free subgroup of finite index in $W$, then $\mathfrak{W} / \Gamma$ is a nonpositively curved Poincaré complex that is not a homology manifold. For simplicity, we restrict our construction to right angled Coxeter groups, which we briefly review below.

Right Angled Coxeter Groups. A simplicial complex $L$ is a flag complex if any complete graph in the 1-skeleton of $L$ is actually the 1-skeleton of a simplex in $L$. The barycentric subdivision of any cell complex is a flag complex; hence, the condition of being a flag complex imposes no restriction on the topology of $L-$ it can be any polyhedron. The importance of flag complexes in CAT(0) geometry stems from the result of Gromov [?, p. 122] that the natural piecewise Euclidean metric on a cubical complex is nonpositively curved (= locally CAT $(0))$ if and only if the link of each vertex is a flag complex.

Suppose $L$ is a finite flag complex. For each integer $k \geq 0$, let $L^{(k)}$ denote the set of $k$-simplices in $L$ and as before let $\mathcal{S}(L)$ denote the poset of all simplices in $L$ (including the empty simplex). 
Associated to $L$ there is a group $W$ defined as follows. For each $i \in L^{(0)}$ introduce a symbol $s_{i}$ and set $S=\left\{s_{i}\right\}_{i \in L^{(0)}}$. $W$ is defined by the presentation:

$$
\left.W=\langle S| s_{i}^{2}=1, s_{i} s_{j}=s_{j} s_{i} \text { when }\{i, j\} \in L^{(1)}\right\rangle .
$$

$(W, S)$ is called a right angled Coxeter system. Its nerve is $L$.

The Cubical Complex $|\mathbf{W}|$. For each $\sigma \in \mathcal{S}(L)$, let $W_{\sigma}$ denote the subgroup generated by the elements of $S$ which correspond to vertices of $\sigma$. Then $W_{\sigma} \simeq$ $\left(\mathbb{Z}_{2}\right)^{\operatorname{dim}(\sigma)+1}$. Set

$$
W \mathcal{S}(L)=\coprod_{\sigma \in \mathcal{S}(L)} W / W_{\sigma}
$$

$W \mathcal{S}(L)$ is called the poset of spherical cosets (the partial order is given by inclusion). The complex $|W|$ is defined to be the geometric realization of $W \mathcal{S}(L)$. There is an obvious left $W$ action on $|W|$. The cubical structure on $|W|$ is defined as follows. There is one vertex of $|W|$ for each element of $W\left(=W / W_{\emptyset}\right)$. For each spherical coset $w W_{\sigma}$, we then fill in a Euclidean cube of dimension $\operatorname{dim}(\sigma)+1$ with vertices corresponding to the elements of $w W_{\sigma}$. (Note that the elements of $W_{\sigma}$ can naturally be identified with the vertices of a cube of dimension $\operatorname{dim}(\sigma)+1$.) The poset of cubes in $|W|$ is $W \mathcal{S}(L)$ and the link of each vertex is $L$.

The geometric realization of the poset $\mathcal{S}(L)$ is denoted $K$. The inclusion $\mathcal{S}(L) \hookrightarrow$ $W \mathcal{S}(L)$ defined by $\sigma \mapsto W_{\sigma}$ induces an inclusion $K \hookrightarrow\left|W_{L}\right|$ and we identify $K$ with its image in $|W|$. Similarly, the orbit projection $W \mathcal{S}(L) \rightarrow \mathcal{S}(L)$ defined by $w W_{\sigma} \mapsto \sigma$ induces a projection $|W| \rightarrow K$ which factors through a homeomorphism $|W| / \mathrm{W} \rightarrow K$. Thus, $K$ is a fundamental domain for the $\mathrm{W}$-action on $|W|$ and the orbit projection $|W| \rightarrow K$ restricts to the identity on $K$.

The geometric realization of $\mathcal{S}(L)_{>\emptyset}$ can be identified with the barycentric subdivision $L^{\prime}$ of $L$. Thus, $K$ is the cone on $L^{\prime}$ (the empty set provides the cone point). For each $i \in L^{(0)}$, let $K_{i}$ denote the geometric realization of $\mathcal{S}(L)_{\geq\{i\}}$, i.e., $K_{i}$ is the closed star of $i$ in $L^{\prime}$. We call $K_{i}$ the mirror of $K$ of type $i$.

Here is another description of $|W|$. For each point $x \in K$ let $\sigma(x)$ be the simplex spanned by $\left\{i \in L^{(0)} \mid x \in K_{i}\right\}$. Then

$$
|W|=(W \times K) / \sim
$$

where the equivalence relation $\sim$ is defined by $(w, x) \sim\left(w^{\prime}, x^{\prime}\right)$ if and only if $x=x^{\prime}$ and $w^{-1} w^{\prime} \in W_{\sigma(x)}$.

For a flag complex $L$ that can be decomposed as $L=L_{1} \cup L_{2}$, we will construct a different CAT(0) cubical complex $\mathfrak{W}$ on which the associated right angled Coxeter group $W$ acts as a cocompact reflection group. The complexes $\mathfrak{W}$ and $|W|$ will have the same pro-homotopy type. However, the topology of the links of vertices in $\mathfrak{W}$ can differ dramatically from that of the links of $|W|$.

The construction of $\mathfrak{W}$. Suppose that a finite flag complex $L$ can be decomposed as the union of two full subcomplexes: $L=L_{1} \cup L_{2}$. Set $L_{0}=L_{1} \cap L_{2}$. Since $L_{0}, L_{1}$ and $L_{2}$ are full subcomplexes of $L$ each of them is a flag complex.

For any simplicial complex $L$ and a point $z$ not in $L$, let $C_{z} L$ be the simplicial complex defined by taking the cone on $L$ with cone point $z$. 
Let $x_{1}, x_{2}$ and $v$ be points that are not in $L$ and define new simplicial complexes:

$$
\begin{aligned}
& \widehat{L}_{1}=L_{1} \cup C_{v} L_{0} \\
& \widehat{L}_{2}=L_{2} \cup C_{v} L_{0} \\
& \widehat{K}_{1}=C_{x_{1}} \widehat{L}_{1} \\
& \widehat{K}_{2}=C_{x_{2}} \widehat{L}_{2}
\end{aligned}
$$

Let $\widehat{K}$ denote the result of gluing $\widehat{K}_{1}$ to $\widehat{K}_{2}$ along $C_{v} L_{0}$.

In Figure ?? we show a simple example that highlights the difference between $K$ and $\widehat{K}$. The original simplicial complex $L$ is a circuit of length 8 , and $K$ is the cone on this octagon. We let $L_{0} \simeq S^{0}$ be two antipodal vertices (indicated by dots in the figure on the right), and let $L_{1}$ and $L_{2}$ be the two simplicial $\operatorname{arcs}$ in $L$ which are separated by $L_{0}$.

Figure 1. The difference between $K$ (left) and $\widehat{K}$ (right)

Returning to the case where $L$ is an arbitrary finite flag complex, we note that $L$ is a subcomplex of $\widehat{K}$ (we think of it as the boundary of $\widehat{K}$ ). Also, $\widehat{K}$ is contractible (it is the union of two contractible pieces glued along a contractible subcomplex). The space $\mathfrak{W}$ is defined by hollowing out each copy of $K$ in $|W|$ and replacing it with a copy of $\widehat{K}$. Since $K$ and $\widehat{K}$ are both contractible, $|W|$ and $\mathfrak{W}$ are proper homotopy equivalent; hence, $\mathfrak{W}$ is also contractible.

Here is a more precise description of $\mathfrak{W}$. Recalling that for $i \in L^{(0)}, K_{i}$ is the closed star of $i$ in the barycentric subdivision of $L$ (which is a subspace of $\widehat{K}$ ), we see that $K_{i}$ is identified with a subspace of $\widehat{K}$. So, define $\widehat{K}_{i}$ to be $K_{i}$. We then proceed as before. For each point $x \in \widehat{K}$, let $\sigma(x)$ be the simplex spanned by $\left\{i \in L^{(0)} \mid x \in \widehat{K}_{i}\right\}$ and let

$$
\mathfrak{W}=(W \times \widehat{K}) / \sim
$$

where the equivalence relation $\sim$ is defined as before.

It is not difficult to define the cubical structure on $\mathfrak{W}$ and to see that it is CAT(0). The vertex set is $W x_{1} \coprod W x_{2}$. For $\alpha=1,2$ and for a spherical coset $w W_{\sigma} \in W \mathcal{S}\left(L_{\alpha}\right)$, the vertices $w W_{\sigma} x_{\alpha}$ span a cube of dimension $\operatorname{dim}(\sigma)+1$. Also, for each spherical coset $w W_{\sigma} \in W \mathcal{S}\left(L_{0}\right)$, we have a cube spanned by $w W_{\sigma} x_{1} \amalg w W_{\sigma} x_{2}$. Its dimension is $\operatorname{dim}(\sigma)+2$. (In particular, corresponding to the case where $\sigma$ is empty, we have an edge from $w x_{1}$ to $w x_{2}$.) For $\alpha=1,2$, the link of $x_{\alpha}$ in $\mathfrak{W}$ is $\widehat{L}_{\alpha}$. Since $\widehat{L}_{\alpha}$ is a flag complex, the cubical structure is $\operatorname{CAT}(0)$. We also note that the punctured link $\widehat{L}_{\alpha}-v$ is homotopy equivalent to $L_{\alpha}$.

Remark. In [?] the above construction was used only in the case where $L$ is a homology sphere and $L_{0} \subset L$ is a homology sphere embedded in codimension one.

In the following examples we will always choose $L$ to be a triangulation of an $n$-sphere and $L_{0}$ to be a codimension one submanifold triangulated as a full subcomplex. $L_{1}$ and $L_{2}$ will then be $n$-manifolds with boundary. (However, $L_{0}, L_{1}$ and $L_{2}$ need not be connected.) Since $L \simeq S^{n},|W|$ is a contractible $(n+1)$-manifold; however, $\mathfrak{W}$ need not be a manifold. 
Example 1. Suppose that $L$ is a 2 -sphere, that $L_{1}$ is an annulus (a collared neighborhood of the equator) and that $L_{2}$ is the disjoint union of the two 2-disks (neighborhoods of the north and south poles). Then $L_{0}=L_{1} \cap L_{2}$ is the disjoint union of two circles, and $\widehat{L}_{1}$ is an annulus with its boundary coned off. So, $\widehat{L}_{1}$ is homeomorphic to a 2 -sphere with two points identified. In particular, the link $\widehat{L}_{1}$ is not simply connected $\left(\pi_{1}\left(\widehat{L}_{1}\right) \simeq \mathbb{Z}\right)$. Similarly, $\widehat{L}_{2}$ is the wedge of two 2 -spheres. The punctured links $L_{1}$ and $L_{2}$ are also not simply connected. Nevertheless, the theorem quoted at the beginning implies that $|W|$ (and hence $\mathfrak{W}$ ) is simply connected at infinity, since the nerve $L$ is a 2 -sphere.

Example 2. Suppose $L$ is an $n$-sphere and $L_{0}$ is a codimension one submanifold separating $L$ into two pieces $L_{1}$ and $L_{2}$. Then $\mathfrak{W}$ is $(n-1)$-connected at infinity by the theorem quoted at the beginning. On the other hand, $\widetilde{H}_{*}\left(\widehat{L}_{1}\right) \simeq H_{*}\left(L_{1}, L_{0}\right)$ can be nonzero in any dimension $<n$. Similarly, the homology of the punctured link $L_{1}$ is fairly arbitrary.

A true optimist might believe that these examples occur because there are two $W$-orbits of vertices, and that if $W$ acts transitively on the 0 -skeleton, then such examples disappear. The following modified version of our construction shows that this speculation is also false.

A construction with only one vertex orbit. Suppose $L_{0}$ is a subcomplex of $L_{1}$ and that $t$ is a simplicial involution on $L_{0}$. Let $L$ denote the result of gluing together two copies of $L_{1}$ along $L_{0}$ via the map $t$. Call the two copies $L_{1}$ and $L_{2}$. Then $t$ extends to an involution on $L$ (also denoted $t$ ) that interchanges $L_{1}$ and $L_{2}$. Let $W$ be the right angled Coxeter group associated to $L$. Let $G$ denote the semidirect product, $G=W \rtimes \mathbb{Z}_{2}$. Here $\mathbb{Z}_{2}$ acts on the vertex set of $L$ (the generating set of $W$ ) via $t$. The $W$-action on $\mathfrak{W}$ extends to a $G$-action. Now there is only one $G$-orbit of vertices.

Example 3. Suppose that $L_{1}$ is the solid torus, $L_{1}=D^{2} \times S^{1}$ and that $L_{0}$ is its boundary, $L_{0}=S^{1} \times S^{1}$. Let $t: S^{1} \times S^{1} \rightarrow S^{1} \times S^{1}$ be the involution which switches the factors. Then $L=S^{3}$ and $\mathfrak{W}$ is 2-connected at infinity. The link of each vertex is isomorphic to $\widehat{L}_{1}$. However, $H_{2}\left(\widehat{L}_{1}\right) \simeq \mathbb{Z}$ and although $\pi_{1}\left(\widehat{L}_{1}\right) \simeq 0$, for the punctured link, $L_{1}$, we have $\pi_{1}\left(L_{1}\right) \simeq \mathbb{Z}$.

Remark. We note that there is a simple method of altering the local topology of $|W|$ so that the connectivity of the links does not coincide with the connectivity at infinity: Form $|W|^{\prime}$ by attaching a copy of $[0,1]$ (or $[0, \infty)$ ) to each vertex of $|W|$. If one attaches unit intervals, then the resulting complex does not have extendable geodesics; if one attaches half lines, then the resulting complex is not cocompact. Further, while the complex $|W|^{\prime}$ deformation retracts onto $|W|,|W|$ does not sit as a retract inside $\mathfrak{W}$.

\section{REFERENCES}

[1] F.D. Ancel, M.W. Davis and C.R. Guilbault, CAT(0) reflection manifolds, in Geometric Topology (Athens, GA, 1993) AMS/IP Stud. Adv. Math., volume 2, Amer. Math. Soc., Providence, RI 1997, pp. 441-445.

[2] N. Brady, J. McCammond, and J. Meier, Local-to-asymptotic topology for cocompact CAT(0) complexes, preprint.

[3] M.W. Davis, Groups generated by reflections and aspherical manifolds not covered by Euclidean space. Ann. of Math. (2) 117 (1983), no. 2, 293-324. 
[4] M.W. Davis, Buildings are CAT(0) in Geometry and Cohomology in Group Theory (Durham, 1994), London Math. Soc. Lecture Note Ser. 252, Cambridge Univ. Press, Cambridge, 1998, pp. 108-123,

[5] M.W. Davis and J. Meier, The topology at infinity of Coxeter groups and buildings, to appear, Comment. Math. Helv.

[6] M. Gromov, Hyperbolic groups, in Essays in Group Theory (ed. S.M. Gersten) M.S.R.I. Publ. 8 Springer-Verlag (Berlin, Heidelberg, New York), 1987, pp. 75-264.

[7] W. Lück, Transformation groups and algebraic K-theory. Lecture Notes in Mathematics 1408, Springer-Verlag, Berlin, 1989.

[8] G. Moussong, Hyperbolic Coxeter groups, PhD thesis, The Ohio State University, 1988.

Department of Mathematics, The Ohio State University, 231 W. 18th Ave, Columbus, $\mathrm{OH} 43210$

E-mail address: mdavis@math.ohio-state.edu

Department of Mathematics, Lafayette College, Easton, PA 18042

E-mail address: meierj@lafayette.edu 